\title{
Letter to the Editor: Experience of Endoscopic Intra-Gastric Balloons Removal During COVID-19 Pandemic in 98 Patients
}

\author{
Nesreen Khidir $^{1}$ (D) $\cdot$ Asaad Salama ${ }^{1} \cdot$ Moataz Bashah $^{1,2}$ \\ Received: 5 September 2020 / Revised: 3 October 2020 / Accepted: 6 October 2020 / Published online: 13 October 2020 \\ (C) Springer Science+Business Media, LLC, part of Springer Nature 2020
}

The novel coronavirus outbreak $(2019-\mathrm{nCoV})$ was declared a Public Health Emergency of International Concern (PHEIC) on 30th January 2020, and a pandemic on 11th March 2020 [1]. International guidelines have been issued, and most elective surgeries were postponed [2]. Additionally, IFSO guidelines recommended postponing the elective Bariatric and Metabolic (B\&M) surgeries [3]. Accordingly, all elective bariatric procedures stopped in our center, and the practice became limited to urgent interventions for bariatric complications.

Gastrointestinal endoscopy is an aerosol-generating procedure (AGP) with a higher risk of SARS-CoV-2 transmission to both patients and medical staff [4]. Nevertheless, bariatric surgery experts agreed that some endoscopic bariatric procedures are semi-elective/urgent procedures and can be performed following specific protocol during this pandemic [5]. Intragastric balloons had been designed to remain in the stomach for 6 months. Delayed intragastric balloon removal is one of the time-sensitive procedures that carry an increased risk of complications, e.g., migration [6]. Two of our patients with overdue intragastric balloons, encountered migration and both presented to emergency with small bowel obstruction. One required laparoscopic surgical exploration to remove the balloon and the other patient successfully passed the balloon with conservative management. Accordingly, avoidance of further complications mandated to structure a practical algorithm to proceed with the removal of the intragastric balloons during the current COVID-19 pandemic.

The proposed algorithm was based on IFSO recommendations for elective surgeries during COVID-19 [5]. It aimed at

Nesreen Khidir

Dr_sora4@hotmail.com

1 Department of Bariatric and Metabolic Surgery, Hamad Medical Corporation, P. O. Box 3050, Doha, Qatar

2 Weill Cornell Medical College, Doha, Qatar protecting the patients and the involved healthcare workers. All patients had general anesthesia (GA) and intubation. Although intubation itself is an AGP, GA was preferred. It minimizes coughing, irritation, and aerosolization that accompany sedation during endoscopy. All patients had air-filled balloons for a minimum of 6 months and were due to removal. Only patients with negative COVID-19 RT-PCR swab tests had the procedure. All procedures were performed following the suggested algorithm (see Fig. 1).

A retrospective review of all patients who underwent endoscopic removal of intra-gastric balloons during COVID-19 pandemic in a tertiary hospital (29 February 2020-15 June 2020). Study endpoints looked at the complications rate of perforation, bleeding, and pancreatitis, the rate of COVID19 infection in patients after endoscopy, and the rate of COVID-19 infection among procedure providers: (two surgeons, two anesthetists, and three nurses). All patients were followed up for 2 weeks after the balloon removal. RT-PCR testing was not repeated routinely after endoscopy unless the patient had symptoms or contact with an infected individual. All the involved staff in removals of intragastric balloon had COVID-19 RT-PCR testing for variable reasons; screening of theater staff or due to contact with other COVID-19 patients. Results were reviewed by the end of June 2020 .

Results of the review revealed ninety-eight patients underwent endoscopic removal of intra-gastric balloons. Patients' age ranged between 14 and 71 years, with a mean of $33.1 \pm 10.9$ years; BMI $32.8 \pm 4.7 \mathrm{~kg} / \mathrm{m}^{2}$. Thirty-four were females (34.7\%). Procedures were performed at $15.6 \pm 7 \mathrm{~min}$. Three patients had T2D (3.1\%), one had HTN (1\%), one was asthmatic (1\%), and one had CAD (1\%). No complications occurred. Patients have been watched for COVID-19 symptoms within 14 days after removal; none developed symptoms while thirteen patients (13.2\%) had RT-PCR swab tests for contact with COVID-19-positive patients and their results were negative. All the involved medical staff in intragastric balloon removals had RT-PCR tests and results were negative by the end of June 2020 . 


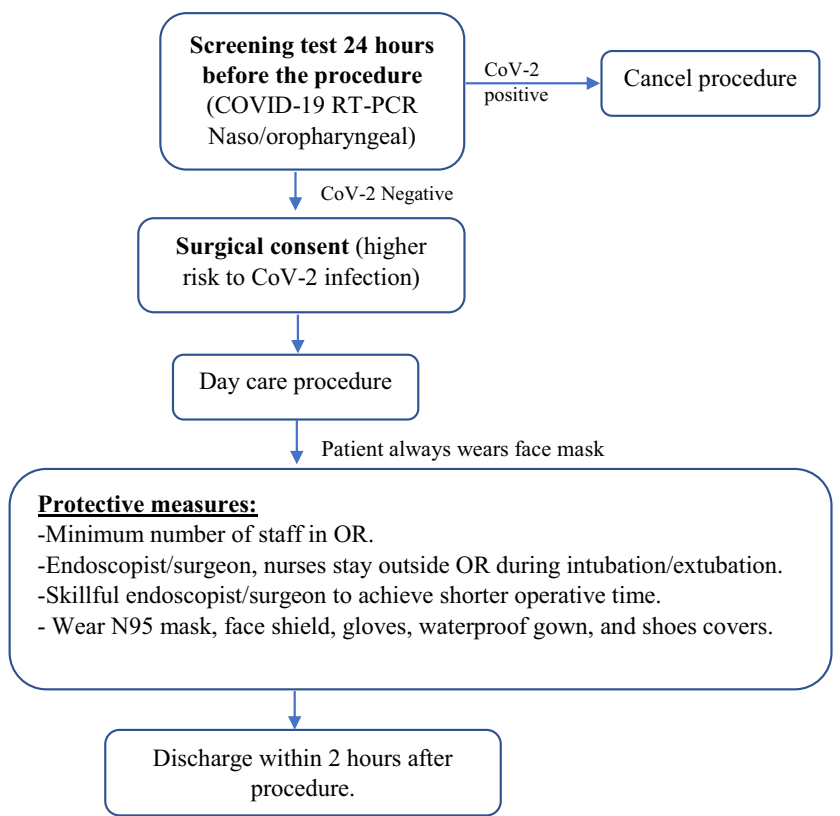

Fig. 1 Algorithm for endoscopic removal of intragastric balloons during COVID-19 pandemic. *All pre- and post-procedure consultations and follow-up were via tele-medicine, reverse-transcription polymerase chain reaction (RT-PCR), coronavirus $(\mathrm{CoV}-2)$

To the best of the authors' knowledge, this article is the first to report and share the experience of endoscopic intra-gastric balloon removal during the era of COVID-19 pandemic. Our reported data has shown that the proposed algorithm is effective in mitigating the risk of infection to both patients and healthcare workers. No post-procedure COVID-19 disease, complications, nor mortality occurred. Several case series of COVID-19 hospitalized patients showed that pre-existing hypertension and diabetes were highly prevalent. Our report included three patients with T2D, one with HTN and one was asthmatic. One had CAD with no reported COVID-19 infection, morbidity, nor mortality.

To comply with social distancing and minimizing the risk of infection, teleconsultations and follow-ups were adopted. Skillful surgeons/endoscopists played an essential role in reducing operative time (15.6 $\pm 7 \mathrm{~min})$. Patients claimed adherence to self-isolation after discharge. However, thirteen patients (13.2\%) had RT-PCR swab tests for contact with COVID-19-positive patients, and their results were negative.
This article concludes that endoscopic intragastric balloons removal during COVID-19 era can be performed safely for both patients and health care providers with a favorable outcome. Authors believe sharing this data will help and reassure some surgeons and endoscopists somewhere out there to proceed with endoscopic removal of intra-gastric balloons in case of future waves of COVID-19 or similar viral pandemics.

\section{Compliance with Ethical Standards}

Conflict of Interest The authors declare that they have no conflict of interest.

Ethical Approval and Informed Consent For this type of study formal consent is not required. The study was approved by the medical research center, Hamad medical corporation. (IRB approval number: MRC-05165).

\section{References}

1. WHO timeline-COVID-19, available at https://www.who.int/newsroom/detail/29-06-2020-covidtimeline. Accessed 29 Jun 2020.

2. COVID-19: Elective Case Triage Guidelines for Surgical Care. Available at https://www.facs.org/covid-19/clinical-guidance/ elective-case/metabolic-bariatric. Accessed 24 Mar 2020.

3. Wang C, Shikora S, Kow L. Recommendations for metabolic and bariatric surgery during the COVID-19 pandemic from IFSO. Obes Surg. 2020;30(6):2071-3. https://doi.org/10.1007/s11695-02004578-1.

4. van Doremalen N, Bushmaker T, Morris DH, et al. Aerosol and surface stability of SARS-CoV-2 as compared with SARS-CoV-1. N Engl J Med. 2020;382(16):1564-7. https://doi.org/10.1056/ NEJMc2004973.

5. L. Angrisani, N. Khidir, G. Prager, J. P. Rafols, M. Suter, et al. (2020) How are we going to restart elective bariatric and metabolic surgery after the peak of COVID-19 pandemic? Sur Res Rep Volume 3(1): 1-5.

6. Sharroufna M, Hassan A, Alabdrabalmeer M, et al. Laparoscopic removal of gastric balloon after failure of endoscopic retrieval. Int J Surg Case Rep. 2019;55:210-2. https://doi.org/10.1016/j.ijscr.2019. 01.043 .

Publisher's Note Springer Nature remains neutral with regard to jurisdictional claims in published maps and institutional affiliations. 